\title{
Capacity of Multi-Antenna Array Systems for HVAC ducts
}

\author{
A.G. Cepni, D.D. Stancil, A.E. Xhafa, B. Henty, P.V. Nikitin ${ }^{\dagger}$, O.K. Tonguz, and D. Brodtkorb \\ Carnegie Mellon University, Department of Electrical and Computer Engineering \\ Pittsburgh, PA 15213, USA \\ $\dagger$ University of Washington, Department of Electrical Engineering \\ Seattle, WA 98195-2500, USA \\ $\ddagger A B B$ Corporate Research, Bergerveien 12, P.O. Box 90 \\ N-1735 Billingstad, NORWAY
}

\begin{abstract}
Multi-element antenna arrays at both transmitter and receiver systems have emerged as a new technique for wireless communication systems in rich multipath environments. HVAC ducts, viewed as over-moded waveguide network systems at $\mathrm{RF}$ frequencies, have the multipath richness through various propagating modes and reflections within the system. This paper studies multiple-input multiple-output (MIMO) systems for HVAC ducts. Computations based on measured channel responses of a 6x6 MIMO system in $0.3 \mathrm{~m}$ diameter HVAC ducts in the 2.4 GHz ISM band demonstrate a five-fold increase in mean capacity over single antenna systems for an SNR of $30 \mathrm{~dB}$.
\end{abstract}

Index Terms - Multi-element array antennas, MIMO capacity, overmoded waveguides, HVAC ducts.

\section{INTRODUCTION}

The increased demand for broadband communications in buildings is bringing new challenges to indoor wireless networks. The challenge is to develop high-speed, mobile, robust, and easy-to-install communication systems. Due to the variations in architecture and types of building materials used, determining the best placement of access points is difficult with conventional wireless LANs. In addition, attenuation through walls and floors limits the range of wireless access points, increasing the number of access points required to achieve adequate coverage. An alternative approach to providing the communication infrastructure in buildings is to recognize that most buildings are already equipped with a microwave distribution system: the heating and ventilation ducts (HVAC). These ducts are designed to carry air to and from all parts of the building, but can also function as hollow waveguides for microwave signals. The concept and preliminary results on this approach have been reported in [1].

An HVAC duct system is electrically isolated from the time-varying indoor environment. Assuming the channel is electrically shielded from the motion of fans and the movement of duct surfaces caused by airflow are negligible, the duct can be modelled as a time-invariant channel. Further, experimental results indicate that the loss in a widely used straight circular HVAC duct with a diameter of $0.3 \mathrm{~m}$ is approximately one tenth of a dB per meter.
An HVAC duct system is a multi-moded complex waveguide network which includes straight ducts with various cross-sections (mostly cylindrical and rectangular), bends, Tjunctions, Y-junctions, tapers, etc. Depending on the geometry of the duct network and the components, the mode content may change along the propagation path. Because of the interference among the modes and the possible reflections from non-uniformities along the propagation path, the channel response exhibits frequency selective behavior resulting in signal dispersion in the time domain. The maximum RMS delay spread of the HVAC channel in our specific case is approximately $300 \mathrm{~ns}$ [2]. This value is between those of typical indoor and outdoor wireless channels.

It has been reported that MIMO techniques may considerably improve the channel capacity of a wireless system under the condition of a low-correlation between the paths relating each transmitting and receiving antenna pair [3],[4]. Such a low correlation is expected in a complex HVAC duct in which modes propagate through the ducts with various propagation characteristics. The fact that the mode content may change through different parts of the duct network also contributes to the de-correlation between the paths. The time-invariant property makes the HVAC duct an ideal environment for the use of multi-antenna array systems.

The use of overmoded waveguides for high capacity communication channels was proposed in the early 1950's [5]. The capacity of a MIMO system was expressed in terms of waveguide modes in [6]. This analysis has been applied to hallways [7] and subway tunnels [8].

In this paper, we provide an analysis of the maximum available capacity with multi-antenna array systems in a HVAC duct channel which can be thought of an overmoded waveguide system.

The remainder of this paper is organized as follows. In Section II, we outline the behavior of a general overmoded waveguide system and its characteristics. The experimental setup is briefly described in Section III. Results and discussions are presented in Section IV, while Section V concludes the paper. 


\section{OVERMODED WAVEGUIDE CHANNEL}

The number of propagating modes in a waveguide depends on its cross-section shape, size, and the excitation frequency. For illustrative purposes, Fig. 1 shows the number of modes in a circular waveguide as a function of the product of operating frequency and the diameter of the waveguide.

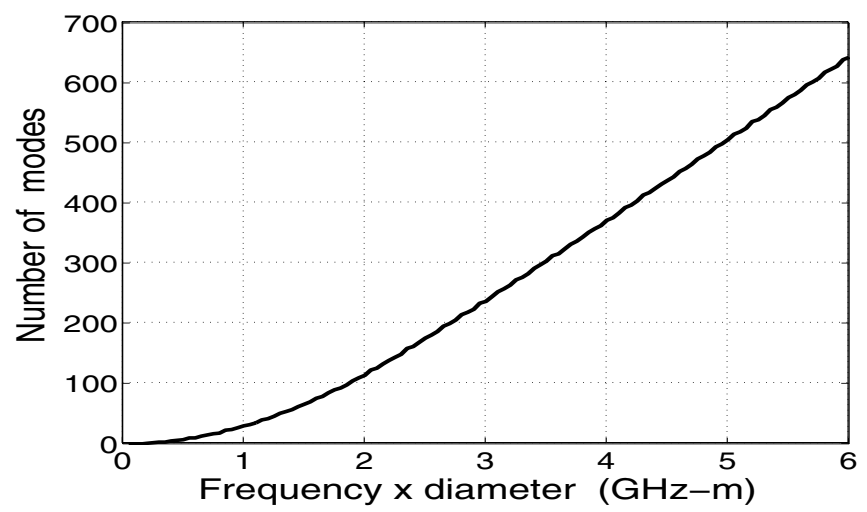

Fig. 1. The number of modes in a circular waveguide as a function of frequency-diameter product.

This plot, for example, shows that for a circular waveguide with a $1 \mathrm{~m}$ diameter, there can exist 643 modes at $6 \mathrm{GHz}$ frequency (frequency $\times$ diameter $=6 \mathrm{GHz}-\mathrm{m}$ ). These modes are like multipath components in a free-space environment. The higher order modes generally experience greater attenuation than lower order modes.

For a typical $0.3 \mathrm{~m}$ diameter duct, the first cut-off frequency is at $576 \mathrm{MHz}$ ( $T E_{11}$ mode), while 17 propagating modes exist in the $2.4 \mathrm{GHz}$ ISM band. As previously mentioned, MIMO techniques work to increase the capacity of the channel under the condition of a low-correlation among various subchannels between the transmitter and the receiver. The capacity of a MIMO channel reaches its maximum value for zero correlation which implies completely independent parallel subchannels. In HVAC ducts, de-correlation is due to the superposition of the numerous modes supported by the structure. The correlation coefficient among various paths becomes lower as the number of propagating modes increases. The complex correlation coefficient between the channel frequency responses of paths $a$ and $b$ is defined as:

$$
\rho=\frac{E\left[a b^{\star}\right]-E[a] E\left[b^{\star}\right]}{\sqrt{\left(E\left[|a|^{2}\right]-|E[a]|^{2}\right)\left(E\left[|b|^{2}\right]-|E[b]|^{2}\right)}} .
$$

where $0 \leq|\rho| \leq 1$.

In a tunnel environment, for example, many modes are excited but only low order modes having lower attenuation can survive after a few hundred meters. A small number of surviving modes causes spatial correlation and can degrade the performance of MIMO systems in a tunnel channel [8].

As previously mentioned, we are interested in calculating the capacity of the HVAC ducts with multi-element antenna arrays. Since the actual received power depends on the distance between transmit and receive antennas, some type of channel normalization is required to facilitate the comparison of the results. Channel normalization is done by scaling the channel matrices such that on average, the power transfer between a single transmit and receive antenna is unity.

Fig. 2 shows the placement of transmit and receive antenna arrays in a generic HVAC duct system. The mode content excited in the duct depends on the antenna type and position. In this paper, we use quarter wavelength monopole antennas which has a center frequency of $2.45 \mathrm{GHz}$. The analysis and experiments were carried out for circular ducts with a diameter of $0.3 \mathrm{~m}$. First, simulation results will be provided for channel properties in straight ducts.

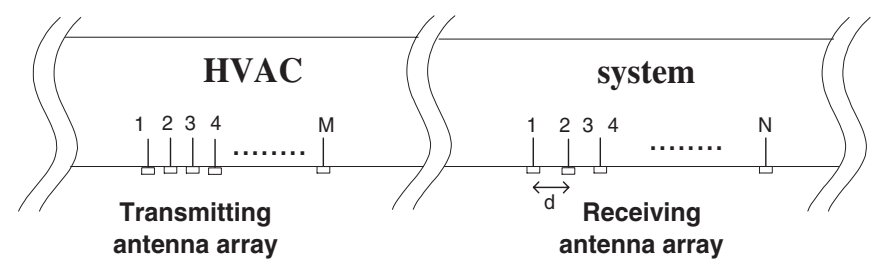

Fig. 2. The placement of transmitter and receiver antenna arrays in an HVAC duct system.

The model presented in [9] was used to generate channel responses between individual pairs of transmit and receive antennas. Channel responses from 2.4-2.5 GHz were generated for antenna spacings from 20 to $200 \mathrm{~m}$, in increments of 2 $\mathrm{cm}$. The ends of the duct were assumed to be terminated in perfect absorbers (Reflection coefficient $=0$ ). At each location, the average value of the signal envelope was computed. The histogram of envelope amplitudes in Fig. 3 shows that the Rayleigh distribution is a good approximation, suggesting that there is no dominant component in the received signal.

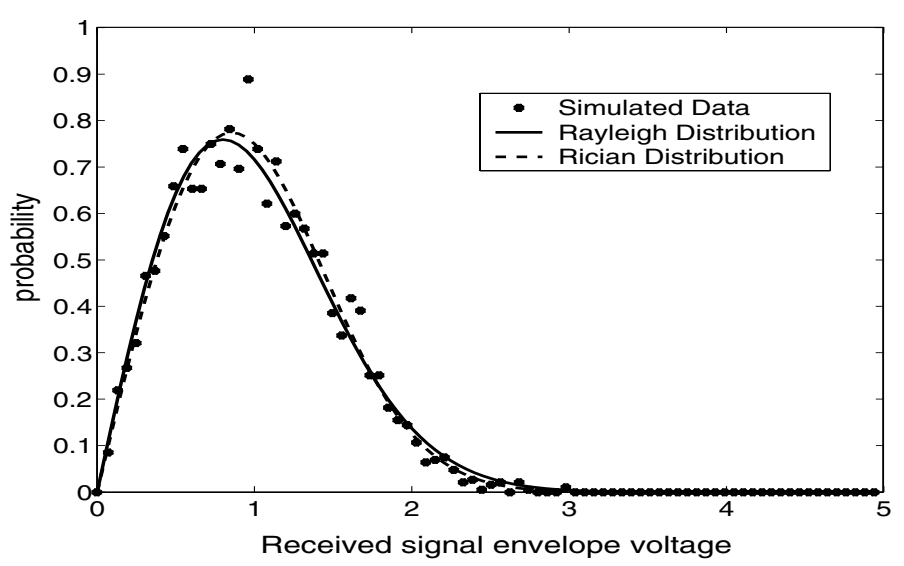

Fig. 3. Signal envelope in a $0.3 \mathrm{~m}$ diameter straight duct with matched terminations at $2.4-2.5 \mathrm{GHz}$ band.

The correlation statistics from the simulations are summarized in Fig. 4. Fig. $4 \mathrm{a}$ and $4 \mathrm{~b}$ show the receiver correlation coefficient as a function of array element and transmitterreceiver spacing, respectively. For Fig. 4a, the receive array element spacing was varied from $0 \mathrm{~m}$ to $2 \mathrm{~m}$ (16 free-space wavelength at $2.45 \mathrm{GHz}$ ) in $1 \mathrm{~cm}$ intervals, with a separation of $20 \mathrm{~m}$ between the nearest transmitting and receiving antenna pair. The minimum array element spacing required for a correlation value below 0.4 is $12 \mathrm{~cm}$ with the perfect absorbers 
at the ends of the duct (see Fig. 4a). The average value of correlation coefficient as a function of distance is plotted in Fig. 4b. Calculations were performed for transmitter-receiver separations from $20 \mathrm{~m}$ to $3000 \mathrm{~m}$ in $20 \mathrm{~m}$ intervals. For each distance, the receiver correlation coefficient was calculated for array element spacings from 0 to $2 \mathrm{~m}$ in $2 \mathrm{~cm}$ intervals. Fig. $4 \mathrm{~b}$ shows the mean value of the correlation coefficient at each distance. As the distance between transmitter and receiver increases, only low-loss modes survive, and the correlation coefficient increases.

Fig. $4 \mathrm{c}$ and $4 \mathrm{~d}$ show the results of repeating the simulation with each end of the duct terminated in a perfectly conducting plate (reflection coefficient $=-1$ ). The behavior of the mean correlation coefficient is very similar to before (of Fig. 4b, d). However, the minimum array element spacing for a correlation coefficient less than 0.4 is reduced to $2 \mathrm{~cm}$ (Fig. 4c).
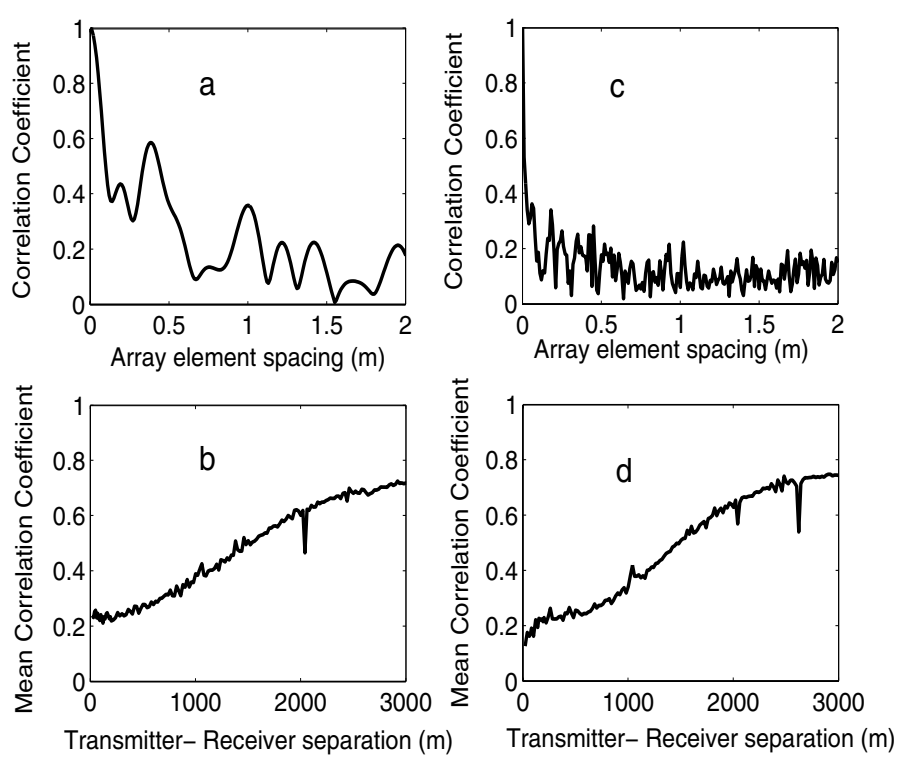

Fig. 4. The receiver correlation coefficient as a function of array element spacing (a), and the mean correlation coefficient as a function of transmitterreceiver separation (b) for perfectly matched terminations. (c) The correlation coefficient vs. element spacing and (d) the mean correlation coefficient vs. transmitter-receiver separation for perfectly reflective terminations.

\section{EXPERIMENTAL SETUP}

To experimentally verify the concept of multi-antenna array systems, the duct network shown in Fig. 5 was constructed. In this experiment, the ends of the duct network were terminated with absorbers to avoid reflections from the surrounding. To illustrate the idea, 6 antennas were placed on the A1 node, and 6 on the A2 node. This formed a $6 \times 6$ MIMO channel between $\mathrm{A} 1$ and A2. Another channel was made using the $3 \times 3 \mathrm{MIMO}$ architecture between B1 and B2. We made 36 measurements for the $6 \times 6$ MIMO channel, and 9 measurements for the $3 \times 3$ case. Since the channel was stationary, no time-averaging was necessary.

The frequency band used in all experiments was 2.4-2.5 $\mathrm{GHz}$, which covers the (2.4-2.4835 GHz) ISM band employed by many popular wireless standards, e.g. IEEE $802.11 \mathrm{~b} / \mathrm{g}$. Measurements were made at 1601 frequency points. The duct components had a diameter of $0.3 \mathrm{~m}$ and were made of galvanized steel. The antennas were monopole probes $3.1 \mathrm{~cm}$ long corresponding to a quarter wavelength at $2.45 \mathrm{GHz}$. The element spacing in the transmit and receive antenna arrays was $5 \mathrm{~cm}$ in all configurations. Although closer than the 12 $\mathrm{cm}$ separation suggested by the spatial correlation calculations with perfect absorbers, our experiments showed that it is still possible to obtain a significant capacity increase with this spacing while reducing the size of the arrays by more than a factor of two. An Agilent E8358A Vector Network Analyzer (VNA) was used for the channel measurements.

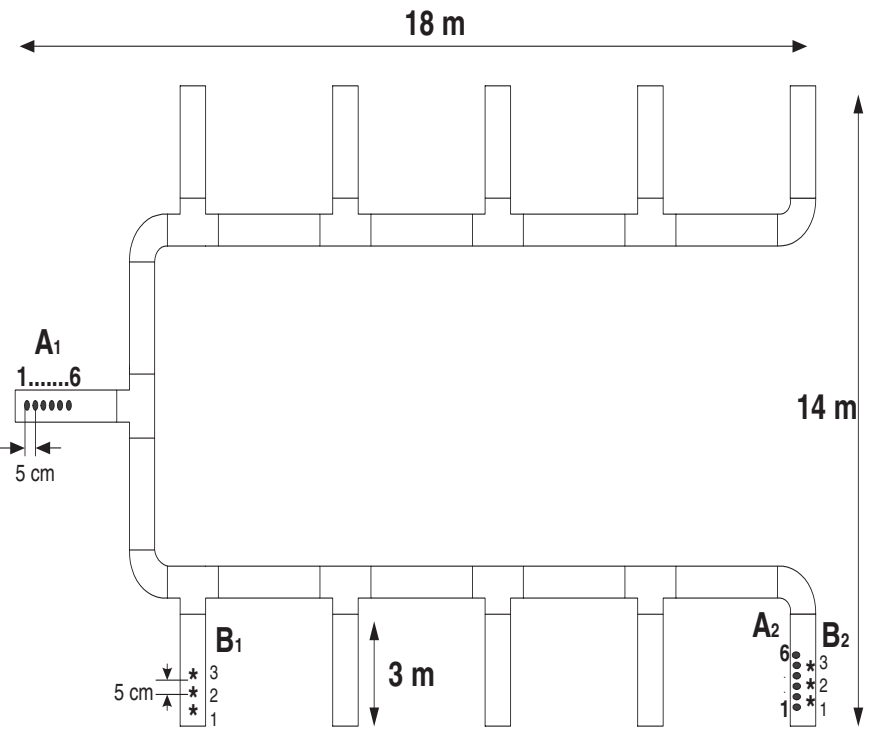

Fig. 5. Experimental setup. A (6,6) MIMO duct channel was formed between $\mathrm{A} 1$ and $\mathrm{A} 2$, and a $(3 \times 3)$ MIMO duct channel was formed between B1 and B2. In all cases, the monopole antennas were separated by $5 \mathrm{~cm}$.

\section{RESUlts AND Discussion}

Fig. 6 shows the normalized path amplitudes for the channel between B1 and B2, and Fig. 7 shows correlation coefficient statistics for the $3 \times 3$ and $6 \times 6$ MIMO channels. The mean value of the correlation coefficient is $0.26 \pm 0.18$ for the paths in the $3 \times 3$ MIMO channel and $0.15 \pm 0.14$ for the paths in the $6 \times 6$ MIMO channel. The low correlation between the various paths in a HVAC duct channel is promising for the application of MIMO techniques to enhance the capacity.

Fig. 8 shows the amplitude PDFs for 3x3 and 6x6 MIMO channels compared with the Rayleigh distribution. The parameters for the Rayleigh distributions are $\sigma^{2}=0.5$ for both configurations. The experimental phase distributions are compared with a uniform distribution for both cases over the interval (-180, 180 degrees).

A close match to the Rayleigh distribution shows that the received signal power is the sum of propagating mode powers that have comparable magnitudes; i.e. there does not appear to be a single dominant path, in agreement with the simulations.

The channel capacity expressed in $\mathrm{bps} / \mathrm{Hz}$ is deduced from the normalized channel matrix $\mathrm{H}$ describing the coupling between each transmitting and receiving antenna. The capacity depends on the correlation between different paths in 


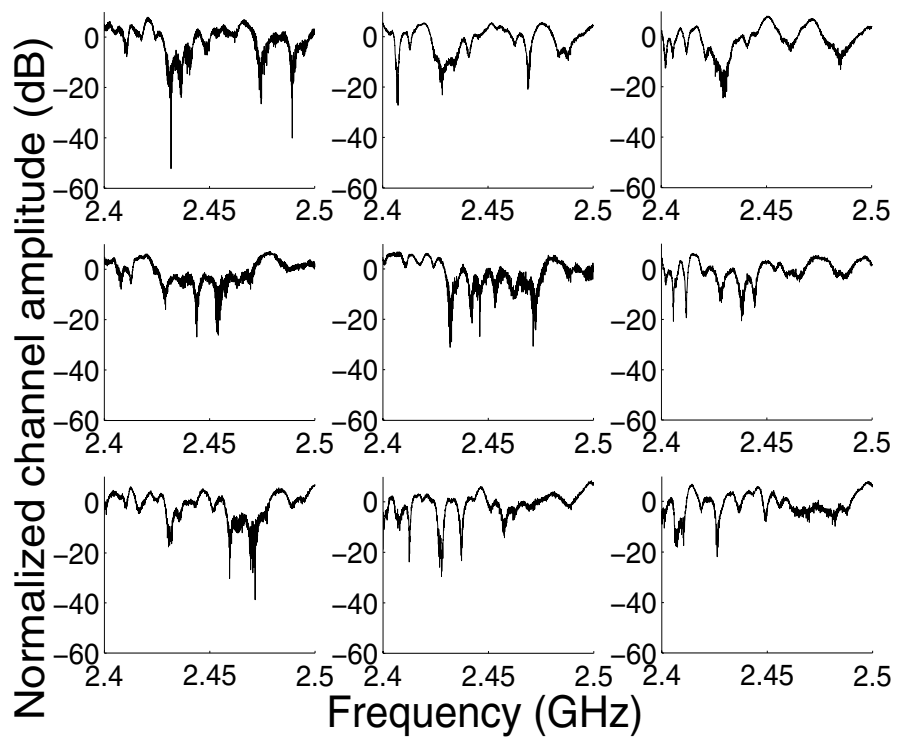

Fig. 6. Normalized path amplitudes in the $3 \times 3$ MIMO channel between B1 and $\mathrm{B} 2$.
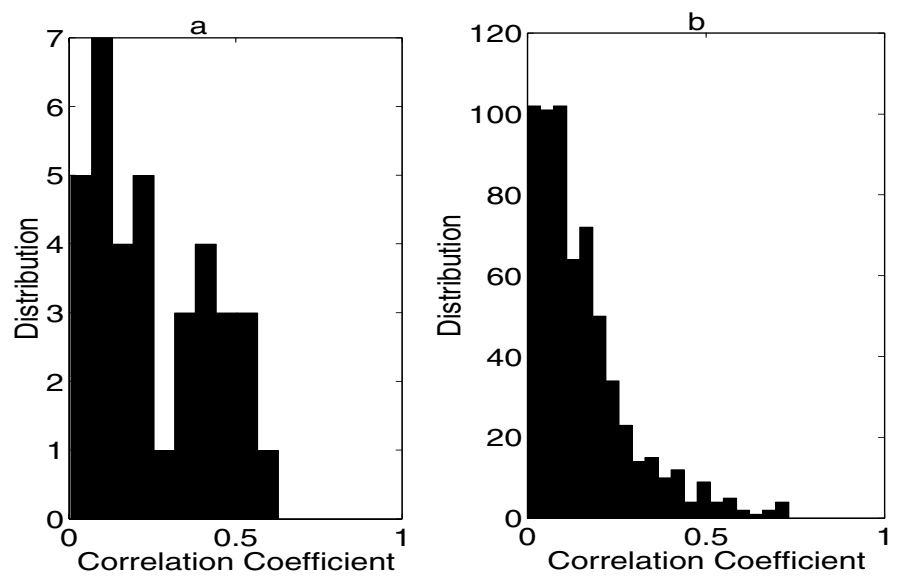

Fig. 7. Statistical distribution of correlation coefficients (a) $3 \times 3$ MIMO channel and (b) 6x6 MIMO channel

the multiple-antenna array system. The expected capacity $C$ expressed in $b p s / H z$, of a random, memoryless MIMO channel in the presence of additive white gaussian noise is given by the generalized Shannon formula [3]:

$$
C=\log _{2}\left[\operatorname{det}\left(I_{n R}+\frac{\rho}{n_{T}} H H^{h}\right)\right] b p s / H z,
$$

where $\rho$ is the average signal to noise ratio per receive antenna, $n_{R}$ is the number of receiving antennas, $n_{T}$ is the number of transmitting antennas, $I_{n}$ is the identity matrix of size $n$ and the upper subscript $h$ means hermitian conjugate of the matrix.

Eqn. 2 assumes that the total transmit power is constant; i.e., for $\mathrm{N}$ antennas the power to each is proportional to $1 / \mathrm{N}$.

We assume a random Rayleigh channel model for which the matrix $\mathrm{H}$ is given by:

$$
H=X+j Y
$$
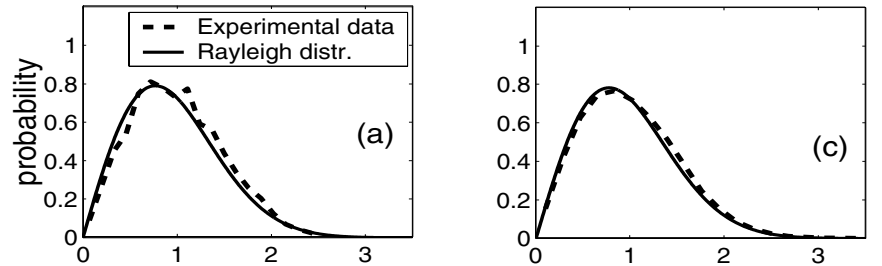

Received signal envelope voltage
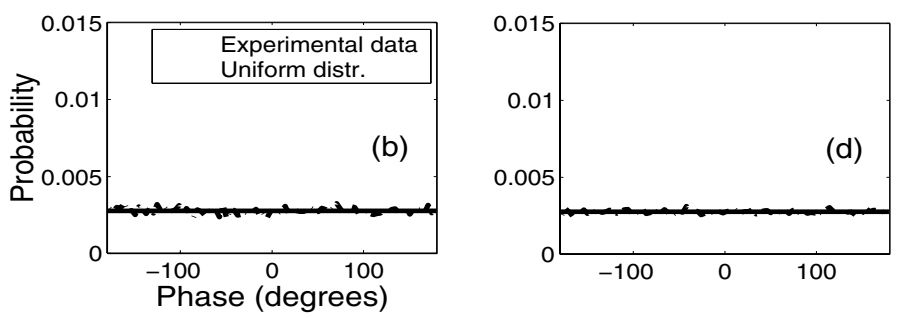

Fig. 8. (a) and (b) Statistical magnitude and phase distributions of HVAC duct channel between $\mathrm{B} 1$ and $\mathrm{B} 2(3 \times 3 \mathrm{MIMO})$. (c) and (d) magnitude and phase distributions between A1 and A2 (6x6 MIMO).

where $X$ and $Y$ are normally distributed random variables with zero mean and $1 / 2$ variance. Thus $E\left[H_{i j}\right]^{2}=1$, and the variance of $\mathrm{H}$ is unity.

Fig. 9 shows the capacities available with different antenna configurations for channels A1-A2 and B1-B2. The notation $(\mathrm{m}, \mathrm{n})$ refers to a system with $\mathrm{m}$ transmit antennas and $\mathrm{n}$ receive antennas. The case $(1, n)$ represents the single input and multiple output (SIMO) configuration, while $(\mathrm{m}, 1)$ represents the multiple input single output (MISO) configuration. The ccdf (complementary cumulative distribution function) graph treats the capacity as a random variable and shows the statistical distribution of the capacity. For the capacity calculations, we have considered 1601 points across $2.4-2.5 \mathrm{GHz}$ band. Compared to the coherence bandwidth of HVAC channel (approximately $4 \mathrm{MHz}$ ), the frequency separation is very small $(62.5 \mathrm{kHz})$. For each frequency point, we calculated the corresponding capacity. The ccdf graph shows the statistical distribution of capacity. In Fig. 9, we assume an SNR of $10 \mathrm{~dB}$ while calculating the capacity from the formula given by Eqn. 2. For the baseline case of single transmit and receive antennas, the capacity is $0.82 \mathrm{bps} / \mathrm{Hz}$ for the channel between $\mathrm{B} 1$ and B2 with 90 percent probability. If we have 3-transmitting antennas at B1 (3-fold transmit diversity), the capacity is increased to $2.3 \mathrm{bps} / \mathrm{Hz}$. With a SIMO system between B1-B2, in which one has 3 -fold receive diversity, the capacity is $3 \mathrm{bps} / \mathrm{Hz}$. With 3 transmit and 3 receive antennas, one can achieve $5.8 \mathrm{bps} / \mathrm{Hz}$ capacity between B1 and B2. As for the channel between A1 and $\mathrm{A} 2$, the capacity is at least $2.5 \mathrm{bps} / \mathrm{Hz}$ with the $(6,1)$ configuration with 90 percent probability, and $4.56 \mathrm{bps} / \mathrm{Hz}$ with the $(1,6)$ architecture. With $10 \mathrm{~dB}$ of SNR, the capacity is $11 \mathrm{bps} / \mathrm{Hz}$ by using 6 transmit, and 6 receive antennas. This shows a substantial improvement in the capacity over the single antenna pair.

The difference between the $(1, n)$ and $(n, 1)$ cases results from holding the total transmit power constant. Under this assumption, the use of $n$ receive antennas results in a factor of $n$ increase in received power, while the use of $n$ transmit antennas does not affect the average total receiver power. 
$\mathrm{SNR}=10 \mathrm{~dB}$
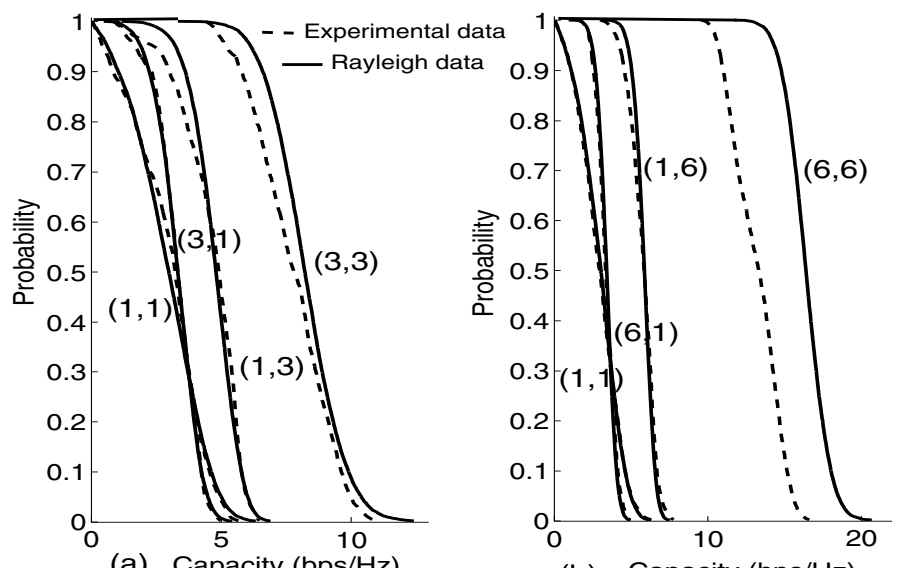

(a) Capacity (bps/Hz)

Fig. 9. CCDF channel capacity with different configurations for the channel between (a) B1-B2, and (b) A1-A2

Fig. 10 shows the average capacity in a MIMO channel for different values of SNR. It has been shown that a wireless system using $\mathrm{n}$ transmitting and $\mathrm{n}$ receiving antennas can achieve a capacity $\mathrm{n}$ times higher than a single-antenna system [3]. In a complex HVAC duct system, the spatial correlation between the antennas in the array system is low. The results show that with $30 \mathrm{~dB}$ of SNR, it is possible to get $24.8 \mathrm{bps} / \mathrm{Hz}$ spectral efficiency by using 3x3 MIMO between B1 and B2. This corresponds to a 2.7 fold capacity enhancement over the baseline case. As for the channel between A1 and A2, with $30 \mathrm{~dB}$ of SNR, $46 \mathrm{bps} / \mathrm{Hz}$ mean capacity is possible using a 6x6 MIMO system. This shows a 5-fold capacity increase for the duct channel.

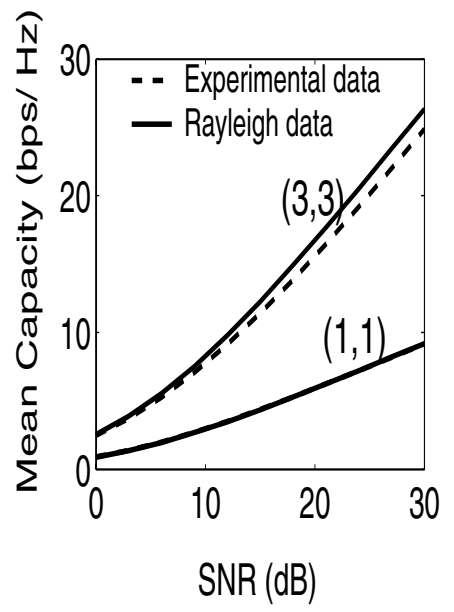

(a)

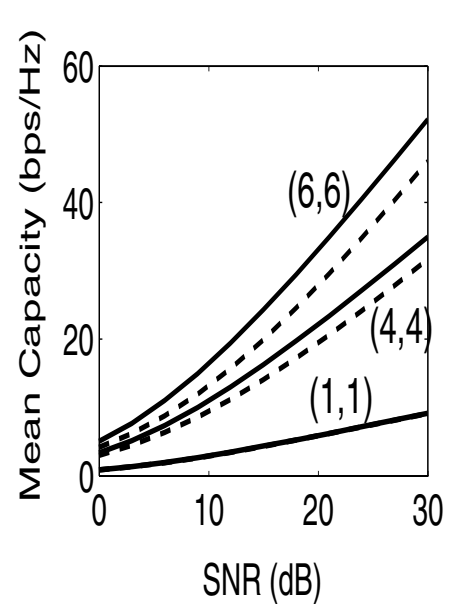

(b)

Fig. 10. Average MIMO channel capacity for different values of SNRs for the channel (a) B1-B2 and (b) A1-A2.

\section{COnCLusions}

In this paper, we presented experimental and analytical results for average HVAC duct channel capacities. The results show that the received signal envelope follows a

Rayleigh distribution in an HVAC channel. In a complex HVAC duct in which modes propagate through the ducts with various propagation characteristics, the correlation among various paths is low, allowing MIMO systems to be applied to the HVAC channel. The results have shown that using $30 \mathrm{~dB}$ of $\mathrm{SNR}$, it is possible to get up to 46 $\mathrm{bps} / \mathrm{Hz}$ average capacity with a $(6 \times 6)$ MIMO architecture. This is a 5-fold capacity increase over the single baseline case.

\section{ACKNOWLEDGEMENT}

This material is based in part upon work supported by the National Science Foundation under Grant No. 0219278.

\section{REFERENCES}

[1] D. D. Stancil, O. K. Tonguz, A. Xhafa, A. Cepni, P. Nikitin, and D. Brodtkorb, "High speed internet access via HVAC ducts: A new approach," in Proc. of IEEE Global Telecomm. Conf. (GLOBECOM'01), vol. 6, pp. 3604-3607, San Antonio, Texas, Nov. 2001.

[2] P.V. Nikitin, D.D. Stancil, O.K. Tonguz, A.E. Xhafa, A.G. Cepni, D. Brodtkorb, "RF propagation in an HVAC duct system: impulse response characteristics of the channel," Antennas and Propagation Society International Symposium, IEEE, vol. 2, 2002 pp. 726-729.

[3] G. J. Foschini and M. J. Gans, "On limits of wireless communication in a fading environment when using multiple antennas," Wireless Personal Commun., vol. 6, no. 3, pp. 311-335, Mar. 1998.

[4] D.S. Shiu, G. J. Foschini and M. J. Gans, and J.M. Kahn, "Fading correlation and its effect on the capacity of multielement antenna systems," IEEE Trans. Com., vol. 48, pp.502-512, March 2000.

[5] S.E. Miller, "Waveguide as a communication medium," Bell System Technical Journal, vol.33(b) pp.1209-1265 (1954).

[6] P. Kyritsi, D.C. Cox, "Expression of MIMO capacity in terms of waveguide modes," Electronics Letters, vol. 38 issue. 18, 2002, pp. $1057-1058$.

[7] P. Kyritsi, D.C. Cox, "Modal analysis of MIMO capacity in a hallway," in Proc. of IEEE Global Telecomm. Conf. (GLOBECOM'01), vol. 1, pp. 567-571, 2001.

[8] A. Lienard, J. Baudet, D. Degardin, P. Degauque, "Capacity of multiantenna array systems in tunnel environment," in Proc. of IEEE Vehicular Technology Conf. (VTC Spring'02), vol. 2, pp. 552-555, 2002.

[9] P. V. Nikitin, D. D. Stancil, A. G. Cepni, O. K. Tonguz, A. E. Xhafa, and D. Brodtkorb, "Propagation model for the HVAC duct as a communication channel," IEEE Transactions on Antennas and Propagation, vol. 51, no 5, pp. 945-951, May 2003. 